\title{
Retraction Note: Thrombendarteriektomie der Arteria carotis und simultane Herzoperation
}

B. Gansera $\cdot$ F. Schmidtler $\cdot$ I. Angelis $\cdot$

J. Weingartner $\cdot$ K. Spiliopoulos $\cdot$ B. M. Kemkes

Published online: 29 November 2012

(c) Springer-Verlag Berlin Heidelberg 2012

Retraction to: Z Kardiol (2004) 93:49-57

DOI 10.1007/s00392-004-1033-9

This article has been retracted as it represents a substantial duplication of the article: B. Gansera, I. Angelis, J. Weingartner, P. Neumaier-Prauser, K. Spiliopoulos, B.M. Kemkes: Simultaneous Carotid Endarterectomy and Cardiac Surgery-Additional Risk Factor or Safety Procedure? Thorac Cardiov Surg (2003) 51:22-27.

The online version of the original article can be found under doi:10.1007/s00392-004-1033-9.

B. Gansera $(\bowtie) \cdot$ F. Schmidtler · I. Angelis · J. Weingartner ·

K. Spiliopoulos - B. M. Kemkes

Krankenhaus München-Bogenhausen, Abteilung für

Kardiovaskularchirurgie, Englschalkingerstr. 77,

81925 München, Germany 